\title{
Effects of oxygen enriched tent by a new oxygen concentration machine on blood oxygen saturation and heart rate in tibet
}

\author{
Guang-Hao Shen ${ }^{1}$, Kun Wang ${ }^{1}$, Li-Hua Lu ${ }^{1}$, Kang-Ning Xie ${ }^{1}$, Qiao-Ling Xu' ${ }^{1}$, Xiao-Ming Wu ${ }^{1}$, \\ Chi Tang ${ }^{1}$, Er-Ping Luo ${ }^{1}$
}

\footnotetext{
${ }^{1}$ Department of Military Medical Equipment \& Metrology, Academy of Biomedical Engineering, the Fourth Military Medical University, Xi'an, China.

Email: ghlulu@fmmu.edu.cn; wangkun6805@163.com
}

Received 19 October 2010; revised 4 December 2010; accepted 12 January 2011.

\begin{abstract}
Many people who live in the low altitude areas are often suffered from hypoxia when they entered the high plateau. This problem may seriously influence the physical and mental state and work efficacy for the travelers and workers. Oxygen enrichment of a small space air at high altitude is considered as a simple way to provide lowlanders enriched oxygen for sleeping and resting, improving work efficiency, so we developed an oxygen concentration machine based on the technology of oxygen enrichment membrane. This paper tested 8 healthy male lowlanders (age 21.63 $\pm 1.77 \mathrm{yr}$ ) who have never exposed to plateau performed an incremental exercise on cycle ergometer at sea-level in order to be used as sea-level controls. Two days later, the same subjects were taken to Lhasa $(3700 \mathrm{~m})$ by air and exposed to the plateau, performed the same exercise as they did at sea-level. The next day, all subjects were asked to enter the experimental tent which was enriched with oxygen (higher than $24 \%$ ) by the oxygen concentration machine and sleep for 10 hours at night, then exposed to plateau and performed the same exercise twice at different time (2 hours and 10 hours after oxygen enrichment). During the tests, subjects must cycled continuously at $60 \mathrm{rpm}$ beginning with a $3 \mathrm{~min}$ exercise intensity of $0 \mathrm{~W}$ followed by incremental increases of $25 \mathrm{~W}$ every $3 \mathrm{~min}$ until $150 \mathrm{~W}$, pulse oxygen saturation $\left(\mathrm{SpO}_{2}\right)$ and heart rate (HR) were recorded. After sleeping in an oxygen enrichment of tent air, 2 hours later, the subjects' load capacity had no difference compared with control group, but significant difference than before (higher $\mathrm{SpO}_{2}$ and lower HR), which indicated that oxygen concentration machine is effective in increasing the oxygen concentration of the air for the tent and sleeping in
\end{abstract}

the oxygen enrichment tent for $10 \mathrm{~h}$ might be effective in improving exercise performance during high-altitude hypoxia. At the same time, 10 hours later, when work-load exceeded $125 \mathrm{~W}$, the same effects were also found. The results indicated the effects of oxygen enrichment of tent air could last a certain period of time.

Keywords: Hypoxia; Oxygen Concentration Machine; Oxygen Enrichment Membrane; High Plateau; Oxygen Enrichment

\section{INTRODUCTION}

Qinghai-Tibet Plateau is called "the roof of the world", has an average elevation of over 4,500 meters, it is the highest plateau of the world. There are over 12 million people in the Qinghai-Tibetan Plateau, of which 6 million are lowlanders (Han culture) and migrants [1,2]. In recent years, with the opening of Qinghai-Tibet railway in China, more and more people who live in low areas enter Qinghai-Tibetan high plateau for science investigation, tour, commercial, or other reasons.

Many of the permanent residents of Qinghai-Tibet Plateau have been living there for generations and have probably benefited from evolutionary adaptation [3]. But for many people who live in the low altitude areas, exposition to plateau often cause them to suffer from hypoxia, and induce acute high altitude disease (AHAD), especially for those with some outdoor works, such as some science investigation and military actions, scientists and soldiers have to sleep in the tent at high altitude without well protection [4]. The most common symptoms of AHAD, including headache, poor appetite, nausea, fatigue, dizziness and insomnia, usually appear within the first three days of arrival at high altitude [5-7]. Moreover, people from low areas may experience 
a decrease in exercise performance on arriving to high altitude. The higher the altitude is, the more critical the situation may occur $[8,9]$. This problem may seriously influence the physical and mental state and work efficacy for the travelers and workers. The basic physiological problem at high altitude is the low partial pressure of oxygen stemming from the reduced barometric pressure $[10,11]$. By raising the concentration of oxygen in the air, the inspired $\mathrm{Po}_{2}$ can be increased and the deleterious effects of the high altitude can be reduced [12]. It has been shown that increasing the oxygen concentration of the air by $1 \%$ (e.g. from 21 to $22 \%$ ) results in a reduction of equivalent altitude of about $300 \mathrm{~m}$ [13]. So oxygen enrichment of a small space air at high altitude is considered as a simple way to provide lowlanders with enriched oxygen and improving their performance [14].

The oxygen enrichment membrane is a kind of gas separation membrane [15], it can be used to enrich oxygen because the oxygen can permeate more than nitrogen, and the oxygen concentration of the air can come up to $40 \%$ through this membrane [16]. It has been widely used in industry, such as steel and chemical, in order to induce the complete combustion and save energy, reduce pollution [17].

This paper first demonstrates small oxygen enrichment space built by tent in Lhasa, and the new oxygen concentration machine based on the technology of oxygen enrichment membrane was used to provide oxygen-enriched gas to the tent. We then report the effects of $10 \mathrm{~h}$ sleeping in such an oxygen enrichment tent on pulse oxygen saturation $\left(\mathrm{SpO}_{2}\right)$ and heart rates $(\mathrm{HR})$ during exercises. We showed that such a machine is effective to provide oxygen-enriched gas and help to build an oxygen enrichment tent conveniently. Oxygen enrichment improves the exercise performance during high-altitude hypoxia, and the effects could last a certain period of time.

This template

\section{MATERIALS AND METHODS}

\subsection{Subjects}

First, Eight young volunteers, all of them are males, normally live at the sea-level regions, with the average age of $21.63 \pm 1.77$ years (aged 19 24), all of Han nationality. All of them do not have the habit of smoking and are healthy without the history of pulmonary, cardiovascular, hematological, renal or hepatic disease, because smoking habit and all of the diseases above may affect the body response to the high altitude. Subjects were all taken to the altitude of $3700 \mathrm{~m}$ by air for the first time. They were familiar with the exercise test on cycle ergometer.

The study was approved by Medical Ethical Committee of Fourth Military Medical University. All the subjects had signed informed consent forms. All variables were measured and groups were separated by the subjects taking exercise at sea-level (control group), before (A1 group) and after oxygen enrichment for 2 hours (A2 group) and 10 hours (A3 group).

\subsection{Oxygen-enriched Tents}

Airtight outdoor tents were placed in Lhasa, which were used as the experimental tents. The tents are all arch shape about $2.05 \times 1.5 \times 1.05 \mathrm{~m}^{3}$, weighted $1.85 \mathrm{kilo}$, made by the material of $210 \mathrm{D}$ nylon and have airtight zippers for people to enter or exit the tents. Each tent is designed for two persons. For 8 subjects, we used 4 tents for them to sleep and 4 oxygen concentration machines were used to provide oxygen-enriched gas to the tents.

\subsection{Ventilation of Tents}

According to the ASHRAE (American Society of Heating, Refrigerating and Air-Conditioning Engineers) standards in 1975 [18], the minimum standard of ventilation of an office, conference room or dormitory was $8.5 \mathrm{~m}^{3}$ per person per hour, that corresponds to 142 $\mathrm{L} / \mathrm{min}$ and is calculated to maintain the carbon dioxide concentration in the room below $0.24 \%$. We use this standard to control the ventilation of the tent in order to maintain the air in the tent acceptable.

For each of the tent has the ability to stay 2 people, the minimum required ventilation is about $300 \mathrm{~L} / \mathrm{min}$. Blowers were used to provide ventilation to the tents through single opening circular ducts of $10 \mathrm{~cm}$ diameter. Each tent has a blower and the duct was put into the tent by a hole we cut on a side of the tent before. The joint was sealed by the rubberized fabric. No exit was provided because the air can find its way out of the tent through random leaks [19].

\subsection{Carbon Dioxide Concentration in the Tents}

Four blood/PH gas analyzers (model 1302, USA) were used to examine the carbon dioxide concentration and $\mathrm{O}_{2}$ concentration of the tent air. Each tent has an analyzer and the output was continuously displayed during subjects sleeping in the tent, in order to evaluate the air and make sure the dioxide concentration is blow $0.24 \%$.

\subsection{Oxygen Concentration Machines}

We developed a new kind of oxygen concentration machine based on the technology of oxygen enrichment membrane. Each machine was about $0.66 \times 0.44 \times 1.2 \mathrm{~m}^{3}$, only required electrical power to drive, it had an outlet 
which could provide oxygen-enriched gas, the outlet could be connected with a circular pipe and the output of the machine was $35 \mathrm{~L} / \mathrm{min}$ of $32 \%$ oxygen. Inside the machine had 12 oxygen enrichment membranes connected with a pump by tubes, the pump was used to pump the air into the tubes at high pressure. The oxygen enrichment membrane is a kind of gas separation membrane, when air passed through these membranes, the oxygen concentration of the air is $35 \%$ and the tubes are injected into one tube which connected the outlet of the machine. The waste air on the other side of the membranes can flow to another tube to the outside environment because of the high pressure.

\subsection{Oxygen Enrichment}

Each tent had an oxygen concentration machine placed outside and the output of the machine was injected into the $10 \mathrm{~cm}$ diameter ventilation duct to the tent. In order to raise the oxygen concentration of the tent, the outlet of the machine was connected with a tube which was injected into the ventilation duct. The blower was first turned off and the oxygen concentration machine was turned on for over $1 \mathrm{~h}$. Blood/PH gas analyzer (model 1302, USA) was used to examine the $\mathrm{O}_{2}$ concentration of the tent air, when the concentration was higher than $25 \%$, turn on the blower so the ventilating air was mixed with the oxygen-enriched gas, which made the $\mathrm{O}_{2}$ concentration of the tent air dropped to $(24.38 \pm 0.25) \%$ and kept steady.

When the subjects slept in the tent, the same ventilation and oxygen-enriched gas were provided and the $\mathrm{O}_{2}$ concentration of the tent air was continuously monitored, and the analyzer showed it was always higher than $24 \%$.

\section{7. $\mathrm{SpO}_{2}$ and $\mathrm{HR}$ Measured}

$\mathrm{SpO}_{2}$ and $\mathrm{HR}$ were recorded by using a multi-parameter monitor (model Philips MP20junior203, USA). When all the subjects arrived in Lhasa, they were asked to have a rest for 2 hours and prepare for the experiment. Then, subjects performed an incremental exercise before sleeping in oxygen enrichment of tent air on cycle ergometer (model EMG-II, China), they cycled continuously at $60 \mathrm{rpm}$ beginning with a $3 \mathrm{~min}$ exercise intensity of $0 \mathrm{~W}$ followed by incremental increases of $25 \mathrm{~W}$ every 3 min until $150 \mathrm{~W} . \mathrm{SpO}_{2}$ and $\mathrm{HR}$ were recorded at every 2.5 minutes during exercise (A1 group).

The next day, when all the subjects were physical recovery, they were asked to enter the tent and sleep in oxygen enrichment of tent air for 10 hours (10:00pm 08:00am). After leaving the room, the same exercise test was performed twice at 10:00am (2 hours after oxygen enrichment, A2 group) and 6:00pm (10 hour after oxy- gen enrichment, A3 group), respectively. And the $\mathrm{SpO}_{2}$ and HR were also recorded at every 2.5 minutes during the two exercises. We compared the two variables between A1 group with A2 group and A3 group, A2 and A3 were also compared with each other.

\subsection{Statistical Methods}

The results were presented as Mean \pm SD and the differences were analyzed by the one-way ANOVA. Statistical analysis was carried out with the SPSS for Windows (version 10.0, SPSS, Inc., Chicago, IL). The level of significance was $P<0.05$.

\section{RESULTS}

\subsection{Feasibility of Oxygen Enrichment of Tent Air}

It was not difficult to set up such an oxygen-enriched tent at high altitude with the oxygen concentration machine. Many kinds of tent had the feasibility of airtight when the zippers were closed. Although pump and the machine were taken to equipped, but they were not big and heavy and could be carried in a car easily. The oxygen concentration of the tent air was always higher than $24 \%$ all the night and the dioxide concentration was blow $0.24 \%$. None of the subjects complained uncomfortable after leaving the tent.

\subsection{Heart Rates (HR) and Blood Oxygen Saturation $\left(\mathrm{SpO}_{2}\right)$ during Exercise}

Heart rates (HR) of subjects both before and after oxygen enrichment increased with incremental exercise intensity. Significant differences were found between A1 and control group from $100 \mathrm{~W}$. $(P<0.05$, Table 1$)$. But, they were not significant difference after enrichment (A2 and A3 group) during a similar stage of exercise when compared with control group. (1) In the A2 group, heart rates were not significant difference at each level; (2) In the A3 group, heart rates were not significantly difference before $150 \mathrm{~W}$ load level.

As expected, the blood oxygen saturation $\left(\mathrm{SpO}_{2}\right)$ after oxygen enrichment was increased significantly. Compared with the control group, average $\mathrm{SpO}_{2}$ was found significantly difference between A1, A2 and A3 group at each level $(0 \sim 150 \mathrm{~W}, P<0.05)$. But Compared with the group before enrichment (A1 group): (1) in the A2 group, the average $\mathrm{SpO}_{2}$ increased significantly after enrichment at each load level $(0 \sim 150 \mathrm{~W}, P<0.05)$. (2) in the A3 group, the average $\mathrm{SpO}_{2}$ was significantly higher since $125 \mathrm{~W}$ load level $(125 \sim 150 \mathrm{~W}, P<0.05)$. And in addition, significant differences were found in $\mathrm{SpO}_{2}$ between A2 and A3 $(0 \mathrm{~W}, 25 \mathrm{~W}, 75 \mathrm{~W}, 100 \mathrm{~W}, 125 \mathrm{~W}, 150$ $\mathrm{W}, P<0.05$, Table 2 ). 
Table 1. Heart rates during exercise before and after oxygen enrichment $(\bar{x} \pm s, \mathrm{n}=8)$.

\begin{tabular}{cccccccc}
\hline \multirow{2}{*}{ Group } & \multicolumn{7}{c}{ Heart rate (beats/min) } \\
\cline { 2 - 7 } & $0 \mathrm{~W}$ & $25 \mathrm{~W}$ & $50 \mathrm{~W}$ & $75 \mathrm{~W}$ & $100 \mathrm{~W}$ & $125 \mathrm{~W}$ & $150 \mathrm{~W}$ \\
\hline Control & $88.76 \pm 2.10$ & $102.13 \pm 4.04$ & $118.22 \pm 4.02$ & $130.71 \pm 2.30$ & $136.79 \pm 2.96$ & $152.18 \pm 2.94$ & $163.28 \pm 1.99$ \\
A1 & $89.00 \pm 2.33$ & $102.63 \pm 4.03$ & $119.00 \pm 3.66$ & $131.25 \pm 2.12$ & $143.88 \pm 3.48^{*}$ & $162.13 \pm 3.87^{*}$ & $174.25 \pm 3.28^{*}$ \\
A2 & $88.88 \pm 2.42$ & $102.75 \pm 5.42$ & $118.50 \pm 4.00$ & $131.38 \pm 3.20$ & $137.88 \pm 3.76$ & $153.63 \pm 3.74$ & $165.38 \pm 2.83$ \\
A3 & $89.88 \pm 2.31$ & $104.38 \pm 5.55$ & $120.25 \pm 3.58$ & $133.25 \pm 3.45$ & $140.13 \pm 5.03$ & $155.00 \pm 3.34$ & $167.88 \pm 2.23^{*}$ \\
\hline
\end{tabular}

Value: Mean \pm SD (standard deviation); Level of significance: $P<0.05$. ${ }^{*} P<0.05$ : as compared with control group by one-way ANOVA

Table 2. $\mathrm{SpO}_{2}$ during exercise before and after oxygen enrichment $(\bar{x} \pm s, \mathrm{n}=8)$.

\begin{tabular}{|c|c|c|c|c|c|c|c|}
\hline \multirow{2}{*}{ Group } & \multicolumn{7}{|c|}{$\mathrm{SpO}_{2}(\%)$} \\
\hline & oW & $25 \mathrm{~W}$ & $50 \mathrm{~W}$ & $75 \mathrm{~W}$ & $100 \mathrm{~W}$ & $125 \mathrm{~W}$ & $150 \mathrm{~W}$ \\
\hline Control & $98.76 \pm 1.05$ & $98.22 \pm 1.11$ & $97.89 \pm 1.91$ & $97.21 \pm 1.88$ & $97.10 \pm 1.67$ & $96.87 \pm 2.32$ & $96.51 \pm 2.10$ \\
\hline A1 & $91.38 \pm 1.06^{*}$ & $90.25 \pm 0.71^{*}$ & $89.00 \pm 1.41^{*}$ & $89.00 \pm 1.20^{*}$ & $88.25 \pm 1.04^{*}$ & $85.75 \pm 0.89^{*}$ & $83.25 \pm 1.04^{*}$ \\
\hline $\mathrm{A} 2$ & $92.88 \pm 0.64^{* \Delta}$ & $91.88 \pm 0.35^{* \Delta}$ & $90.63 \pm 0.52^{* \Delta}$ & $90.13 \pm 0.83^{* \Delta}$ & $89.38 \pm 0.92^{* \Delta}$ & $87.88 \pm 0.83^{{ }^{* \Delta}}$ & $85.75 \pm 1.04^{* \Delta}$ \\
\hline A3 & $91.13 \pm 0.83^{\text {厺 }}$ & $90.63 \pm 0.52^{\text {ㅎ }}$ & $89.88 \pm 1.13$ & $89.21 \pm 1.20^{\text {名 }}$ & $87.63 \pm 0.92^{\text {하 }}$ & $86.63 \pm 0.74^{\sqrt{4} \Delta}$ & 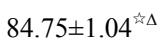 \\
\hline
\end{tabular}

Value: Mean $\pm \mathrm{SD}$ (standard deviation); Level of significance: $P<0.05 . * P<0.05$ : as compared with control group by one-way ANOVA; ${ }^{\wedge} P<0.05:$ A2, A3 compared with A1 by one-way ANOVA. ${ }^{\star} P<0.05$ : A 3 compared with A2 by one-way ANOVA.

\section{DISSCUSSION}

\subsection{Feasibility and Costs of Oxygen Concentration Machines}

We used the oxygen concentration machines to provide the oxygen-enriched gas to the tents because they were convenient, and the costs for these machines were low. For the machines were based on the technology of oxygen enrichment membranes, the oxygen-enriched gas could be continuously obtained by the environment air at high altitude without changing the oxygen enrichment membranes. Each machine was about $0.66 \times 0.44 \times 1.2 \mathrm{~m}^{3}$ and weighted about $30 \mathrm{~kg}$, which could be easily carried to outdoors by a car.

Each machine required electrical power to drive; the car electrical power can perfectly provided the power in the open plateau. The power consumption of each machine was just $600 \mathrm{~W}$ and that cost about $¥ 0.3$ an hour. So it is a very convenient and practical way to build an oxygen enriched tent with such a machine with very low costs.

\subsection{Feasibility of oxygen enriched tents}

We found that oxygen enriched tents were very useful and convenient to solve the problem of hypoxia for outdoor workers at high altitude, especially in some remote areas. For now, most of the oxygen enriched ideas were based on rooms, which meant an airtight room was needed; the room may cost much and could not be moved. The oxygen enriched tents could be set up wherever needed and the cost of a tent must be much lower than a room.

In this paper, we used a blower and a single opening circular duct of $10 \mathrm{~cm}$ diameter for each tent to provide ventilation, that total costs of these were low too. We studied 4 arch shape tent, each of them was about $2.05 \times 1.5 \times 1.05 \mathrm{~m}^{3}$ with 2 persons sleeping inside for 10 hours, with an oxygen concentration machine provided oxygen-enriched gas, the oxygen concentration of the tent air was always higher than $24 \%$ and the carbon dioxide concentration was lower than $0.24 \%$, meet the ASHRAE (American Society of Heating, Refrigerating and Air-Conditioning Engineers) standards.[20]

\subsection{Heart Rates (HR) and Blood Oxygen Saturation $\left(\mathrm{SpO}_{2}\right)$ Studies during Exercises}

In this paper, we compared the control group's heart rates and blood oxygen saturation $\left(\mathrm{SpO}_{2}\right)$ with before (A1 group) and after oxygen enrichment for 2 (A2 group) and 10 hours (A3 group) during the exercises at each load. Heart rates reflect the cardiac function of body. If the heart beats too fast that means the work load will not be suitable for people to continue and a short time of rest will be needed. When people work at altitude, the working heart rates can reflect the ability to work and load capacity.

Compared with control group, heart rates were found significantly higher since $100 \mathrm{~W}$ load level $(100 \sim 150 \mathrm{~W}$, $P<0.05)$ at A1, but no differences were found between A2 group at each level, which showed oxygen enrichment for 10 hours could significant increase the ability to work and load capacity at high altitude to the work and load capacity at sea level. A3 group's heart rates were not significantly difference before $150 \mathrm{~W}$ load level compared with control group, which showed that the increased ability to work and load capacity could last a certain period of time (Figure 1). 


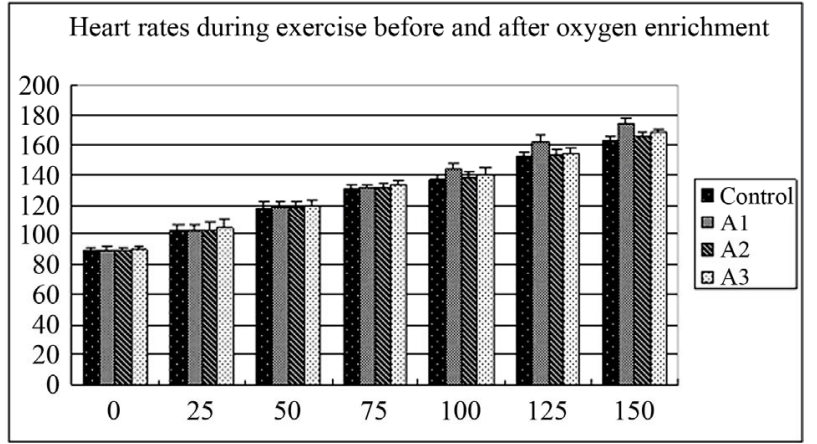

Figure 1. Heart rates during exercise, at the level of significance: $P<0.05$.

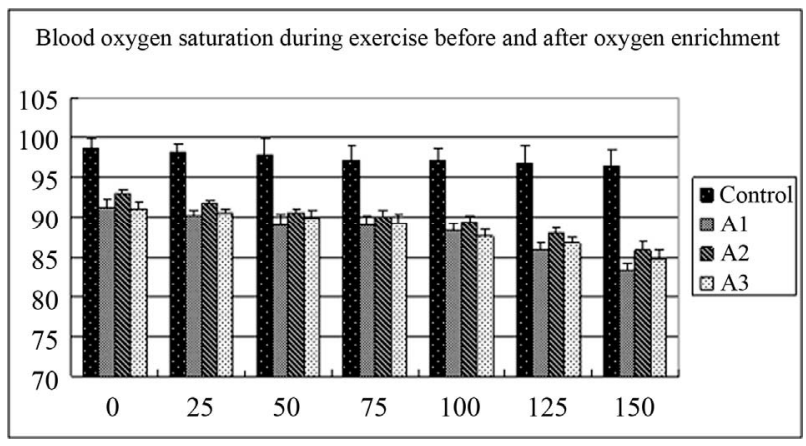

Figure 2. Blood oxygen saturation $\left(\mathrm{SpO}_{2}\right)$ during exercise, at the level of significance: $P<0.05$.

$\mathrm{SpO}_{2}$ is an important indicator of oxygen supply for the body. At high altitude, the low partial pressure of oxygen stemming from the reduced barometric pressure caused less oxygen diffuse into the capillaries within the lungs, finally caused hypoxia and low $\mathrm{SpO}_{2}$ [21].

In this paper, we found control group had significant higher $\mathrm{SpO}_{2}$ at each load level $(0 \sim 150 \mathrm{~W}, P<0.05)$ compared with the other groups during the exercise. A2 group had significant higher $\mathrm{SpO}_{2}$ compared between $\mathrm{A} 1$ $(0 \sim 150 \mathrm{~W}, P<0.05)$, A3 group had the same effects since $125 \mathrm{~W}(125 \sim 150 \mathrm{~W}, P<0.05)$, that showed oxygen-enriched of tent air raising the concentration of oxygen in the air, inspired Po2 can be increased by this, so more oxygen diffuse into the capillaries within the lungs, and the deleterious effects of the high altitude can be reduced (Figure 2).

In this paper, we also found significant differences with $\mathrm{SpO}_{2}$ between $\mathrm{A} 2$ and $\mathrm{A} 3$ group at some load level $(0 \mathrm{~W}, 25 \mathrm{~W}, 75 \mathrm{~W}, 100 \mathrm{~W}, 125 \mathrm{~W}, 150 \mathrm{~W}, P<0.05)$. Further studies should be needed to analyze the differences and expound the underlying mechanism.

\section{ACKNOWLEDGEMENTS}

The authors would like to thank the director of the Department of Academy of Biomedical Engineering, the Fourth Military Medical
University, for providing necessary support for this study. And the 8 volunteers for their working during the whole study.

\section{REFERENCES}

[1] West, J.B. (1986) Highest inhabitants in the world. Nature, 324, 517.

[2] Wu, T, Kayser, B. (2006) High altitude adaptation in Tibetans. High Altitude Medicine \& Biology, 7, 193-208.

[3] Luks, A.M, van Melick, H., Batarse, R.R., Grant I. and West J.B. (1998). Room oxygen enrichment improves sleep and subsequent day-time performance at high altitude. Respiration Physiology, 113, 247-258.

[4] Siqués,P. and Brito, J. (2009) Blood Pressure Responses in Young Adults First Exposed to High Altitude for 12 Months at 3550m. High Altitude Medicine \&Biology, 10, 329-335.

[5] Ha, Z.D., He., T.H., Zhang, X.Z., Wang, W., Ma, Y. and Jian, X.Q. (2004) Effects of oxygen enrichment of room air on sleep patterns at high altitude. Zhonghua Nei Ke Za Zhi, 43, 368-370.

[6] Sato, M., Severinghaus, J.W., Powell, F.L., Xu, F.D. and Spellman, M.J (1992) Augmented hypoxic ventilatory response in men at altitude. Journal of Applied Physiology, 73, 101-107.

[7] Sato, M., Severinghaus, J.W. and Bickler, P. (1994) Time course of augmentation and depression of hypoxic ventilatory responses at altitude. Journal of Applied Physiology, 77, 313-316.

[8] Calbet, J.A. (2003) Chronic hypoxia increases blood pressure and noradrenaline spillover in healthy humans. The Journal of Physiology, 551, 379-386.

[9] Black, C.P., Tenney, S.M. (1980) Oxygen transport during progressive hypoxia in high-altitude and sea-level waterfowl. Respiration Physiology. 39, 217-239.

[10] West J.B. (2003) Improving oxygenation at high altitude: acclimatization and $\mathrm{O} 2$ enrichment. High Altitude Medicine \&Biology, 4(3): 389-398.

[11] Fletcher, E.C. (2001) Invited review: physiological consequences of intermittent hypoxia: systemic blood pressure. Journal of Applied Physiology, 90, 1600- 1605.

[12] West, J.B. (2002) Potential use of oxygen enrichment of room air in mountain resorts. High Altitude Medicine \& Biology, 3, 59-64.

[13] West, J.B. (1995) Oxygen enrichment of room air to relieve the hypoxia of high altitude. Respiration Physiology, 99, 225-232.

[14] West, J.B. (2007) Adventures in high-altitude physiology. Advances in Experimental Medicine Biology, 588, 7-16.

[15] Richard, W.B. (2002) Future Directions of Membrane Gas Separation Technology. Industrial \& Engineering Chemistry Research, 41, 1393-1411.

[16] Rigby, G.R. and Watson, H.C. (1994) Application of membrane gas separation to oxygen enrichment of diesel engines. Journal of Membrane Science, 87, 159-169.

[17] Byuna, Hongsik., Honga, B. and Lee, B. (2006) The effect of oxygen enriched air obtained by gas separation membranes from the emission gas of diesel engines. Desalination, 193, 73-81 
[18] ASHRAE Standard 90-75. (1975) Energy Conservation in New Building Design, Atlanta, GA: American Society of Heating, Refrigerating, and Air-Conditioning Engineers, Inc.

[19] Luks, A.M., van Melick H., Batarse R.R., Grant I. and West, J.B. (1998) Room oxygen enrichment improves sleep and subsequent day-time performance at high altitude. Respiration Physiology, 113, 247-258.
[20] ASHRAE Standard 62-1989. (1989) Ventilation for Acceptable Indoor Air Quality. Atlanta, GA: American Society of Heating, Refrigerating and Air-Conditioning Engineers, Inc.

[21] Cui, J.H., Zhang, X.Z., Zhang, J.L., Zhang, F., Ma, Y. and Wang, D.W. (2003) Effects of enriched oxygen on human free radical metabolism during exercise at 5380 m plateau. Space Med Med Eng (Beijing), 16, 377-378. 